\title{
The anomalous mass dimension from the techniquark propagator in Minimal Walking Technicolor
}

Daniel August ${ }^{* \dagger}$

Friedrich-Schiller-University Jena

Max-Wien-Platz 1, D-07743 Jena, Germany

E-mail: daniel.august@uni-jena.de

\section{Axel Maas}

Friedrich-Schiller-University Jena

Max-Wien-Platz 1, D-07743 Jena, Germany

E-mail: axelmaas@web.de

\begin{abstract}
Minimal Walking Technicolor is a 2-color QCD-like theory with two quark flavors in the adjoint representation. We calculated the techniquark propagator in minimal Landau gauge, using lattice simulations of the standard Wilson action. This data enabled us to derive the anomalous mass dimension from a single lattice setup. Furthermore we calculated the respective Schwinger functions.
\end{abstract}

31st International Symposium on Lattice Field Theory LATTICE 2013

July 29 - August 3, 2013

Mainz, Germany

\footnotetext{
* Speaker.

${ }^{\dagger}$ Supported by the DFG graduate school 1523-1 and under grant number MA3935/5-1

${ }^{\ddagger}$ Supported by the DFG under grant number MA3935/5-1
} 


\section{Introduction}

The Standard Model Higgs is a fundamental scalar particle which is responsible for the electroweak symmetry breaking and the mass generation of the Standard Model particles. Calculating the quantum corrections to its own mass one finds [1]

$$
M_{H_{\text {rem }}}^{2}-M_{H}^{2} \sim \Lambda^{2}
$$

where $\Lambda$ is the cutoff of the Standard Model. Since this cutoff could go up to the Planck scale we have a fine tuning problem for the Higgs mass. To get rid of this problem, several possible extensions to the Standard Model were proposed [1,2]. One of these is Technicolor(TC) which replaces the Higgs particle by a bound state of techniquarks. The interaction between these new particles is supposed to be QCD-like which also introduce technigluons. Furthermore one has to introduce new mechanisms to comply with phenomenology.

One proposal is the Minimal Walking Technicolor(MWT) [1]. The TC sector of this theory uses the gauge group $\mathrm{SU}(2)$ and has two techniquarks in the adjoint representation and one technigluon. Furthermore one has to introduce an extra pair of leptons. Since this theory is walking one finds [1]

$$
\langle\bar{Q} Q\rangle_{E T C} \sim\left(\frac{\Lambda_{E T C}}{\Lambda_{T C}}\right)^{\gamma}\langle\bar{Q} Q\rangle_{T C}
$$

where $\gamma$ is the anomalous mass dimension of the techniquark propagator. As one sees this quantity could enhance the chiral condensate on the Extended Technicolor(ETC) scale which in turn is responsible for the masses of the Standard Model quarks. If we compare our theory to the observed quark masses we find that $\gamma$ should be of the order of one [1]. Yet there are a lot of results suggesting that this quantity is substantially smaller $[3,4,5]$. Since these results used indirect methods, our aim is to determine it directly by calculating the techniquark propagator.

The quark propagator is a gauge dependent quantity, therefore we will fix our gauge to minimal Landau gauge. We implement the method described in [6]. On the other hand the anomalous mass dimension is a gauge independent quantity. For this reason the gauge fixing procedure will not influence our final result.

\section{Calculation}

All results presented in this paper were created using configurations provided by the authors of [3, 7, 8, 9]. For details concerning their creation and properties see [9]. One important note is that we expect a quasi-conformal behavior in the chiral limit [1]. Furthermore only configurations for $\beta=2.25$ with only a limited amount of lattice sizes were available. This limits the analysis of the systematic errors.

The quark propagator in minimal Landau gauge in the continuum is parametrized by the scalar functions $\mathrm{A}, \mathrm{B}, \mathrm{M}$ and $\mathrm{Z}$

$$
\begin{array}{r}
S^{a b}(p)=\delta^{a b} \frac{A\left(p^{2}\right) \gamma_{\mu} p_{\mu}+B\left(p^{2}\right)}{A\left(p^{2}\right)^{2} p^{2}+B\left(p^{2}\right)^{2}}=\delta^{a b} Z\left(p^{2}\right) \frac{i \not p+M\left(p^{2}\right)}{p^{2}+M\left(p^{2}\right)^{2}} \\
Z\left(p^{2}\right)=\frac{1}{A\left(p^{2}\right)}, \quad M\left(p^{2}\right)=\frac{B\left(p^{2}\right)}{A\left(p^{2}\right)}
\end{array}
$$


where $\gamma_{\mu}$ are the Euclidean Dirac matrices, $\mathrm{Z}$ is the wave-function renormalization and $\mathrm{M}$ is the mass function. Using the latter parameters we can define the Schwinger functions [10,11]

$$
\begin{aligned}
& \Delta_{V}=\frac{1}{\pi} \int_{0}^{\infty} d p \cos (t p) \frac{Z\left(p^{2}\right)}{p^{2}+M\left(p^{2}\right)^{2}} \\
& \Delta_{s}=\frac{1}{\pi} \int_{0}^{\infty} d p \cos (t p) \frac{Z\left(p^{2}\right) M\left(p^{2}\right)}{p^{2}+M\left(p^{2}\right)^{2}}
\end{aligned}
$$

where $v$ refers to the vector part and $\mathrm{s}$ to the scalar part. The functions enable us to determine a possible pole mass.

To calculate any of the before mentioned functions we use Wilson fermions

$$
D(x, y)=-\frac{1}{2} \sum_{\mu}\left(1-\gamma_{\mu}\right) U_{\mu}^{a} \delta_{x+\mu y}+(m+4) \delta_{x y}
$$

where $U_{\mu}^{a}$ are link variables in the adjoint representation. We retrieve these from the links in the fundamental representation by

$$
U_{\mu b c}^{a}=\frac{1}{2} \operatorname{tr}\left(\sigma^{b} U_{\mu}^{\dagger} \sigma^{c} U_{\mu}\right)
$$

with the Pauli matrices $\sigma^{a}$. To obtain the quark propagator we have to invert and Fourier-transform the Dirac operator $D(x, y)$ for the Wilson fermions. The inversion is done with a standard biconjugate gradient inversion while we only use momenta in time direction for the Fourier-transformation to make full use of the extended time direction, for details see [12]. Afterwards we apply lattice corrections which ensure that we retrieve the continuum results for the free case. The code has been thoroughly checked for the free case.

After this procedure we have to renormalize the A,B,Z and $\mathrm{M}$ functions. We have chosen the following scheme

$$
S(p=\mu)=\frac{1}{-i \not p+m_{q}}
$$

The only question remaining is the value of $m_{q}$. This problem is quite important because there is no experimental data available for $m_{q}$. One possibility would be to chose the bare mass but this quantity becomes negative if we go to the chiral limit. For this reasons we have chosen the PCAC mass $m_{q}=m_{P C A C}$ given for some configurations in [3]. To get access to the remaining ones we used linear extrapolation/interpolation.

Finally we have to set the scale. This task was already done in a preceding work investigating technigluonic two-point and three-point functions [13]. In this work the mass of the scalar glueball is set to $2 \mathrm{TeV}$. Following [14, 15] this state mixes with or is the Higgs. Therefore in light of the recent LHC results [16, 17], we changed this procedure to get a Higgs mass of $125 \mathrm{GeV}$. To avoid possible problems with this scale setting procedure we will present our results also in lattice units.

\section{Mass function}

To derive the anomalous mass dimension from the quark propagator we have to use a fit function which describes the mass function. Considering observations from the quenched calculation 


\begin{tabular}{|c|c|c|c|c|c|c|c|c|c|}
\hline$a m_{0}$ & $N_{s}$ & $a m_{\mathrm{PCAC}}$ & $m_{\mathrm{PCAC}}$ & $2 \mathrm{~b}$ & $\gamma_{f}$ & $a M(0)$ & $M(0)$ & $-a\langle\bar{\Psi} \Psi\rangle^{\frac{1}{3}}$ & $-\langle\bar{\Psi} \Psi\rangle^{\frac{1}{3}}$ \\
\hline 0.5 & 16 & 2.02 & 214 & $0.18(2)$ & $0.967(6)$ & $2.22(5)$ & $235(6)$ & $0.691(2)$ & $73.2(3)$ \\
\hline 0.25 & 16 & 1.74 & 188 & $0.18(2)$ & $0.972(8)$ & $1.9(2)$ & $205(22)$ & $0.656(3)$ & $70.9(4)$ \\
\hline 0 & 16 & 1.46 & 161 & $0.18(2)$ & $0.980(8)$ & $1.7(1)$ & $187(11)$ & $0.618(2)$ & $68.0(3)$ \\
\hline-0.25 & 16 & 1.18 & 133 & $0.17(2)$ & $0.991(7)$ & $1.41(2)$ & $159(3)$ & $0.573(1)$ & $64.7(2)$ \\
\hline-0.5 & 16 & 0.897 & 108 & $0.15(2)$ & $0.99(2)$ & $1.09(3)$ & $131(4)$ & $0.517(7)$ & $62.0(9)$ \\
\hline-0.75 & 16 & 0.617 & 83.9 & $0.11(2)$ & $0.97(2)$ & $0.78(7)$ & $106(10)$ & $0.446(5)$ & $60.7(7)$ \\
\hline-0.9 & 16 & 0.449 & 68.2 & $0.06(3)$ & $0.96(2)$ & $0.50(7)$ & $76(11)$ & $0.41(1)$ & $62(2)$ \\
\hline-0.95 & 16 & 0.393 & 62.1 & $0.01(7)$ & $0.93(6)$ & $0.51(17)$ & $81(28)$ & $0.37(2)$ & $58(4)$ \\
\hline-0.95 & 24 & 0.393 & 62.1 & $0.02(3)$ & $0.96(2)$ & $0.46(6)$ & $73(10)$ & $0.38(1)$ & $60(2)$ \\
\hline-0.975 & 16 & 0.365 & 62.8 & $0.04(11)$ & $0.89(11)$ & $0.34(18)$ & $58(31)$ & $0.36(2)$ & $62(4)$ \\
\hline-1 & 24 & 0.337 & 64.0 & $0.00(2)$ & $0.97(2)$ & $0.35(6)$ & $67(11)$ & $0.366(6)$ & $70(2)$ \\
\hline-1.05 & 24 & 0.277 & 67.9 & $0.02(4)$ & $1.00(3)$ & $0.26(5)$ & $64(13)$ & $0.346(6)$ & $85(15)$ \\
\hline
\end{tabular}

Table 1: Fit parameters for the techniquark mass function. Dimensionful units are given in GeV.

in [12] we have to consider at least two different possibilities. The first is the mass function for the chiral symmetric case

$$
M\left(p^{2}\right)=M(\mu)\left(\omega \ln \frac{p^{2}}{\mu^{2}}+1\right)^{-\gamma}
$$

where $\gamma$ is the mass anomalous dimension. On the other hand we looked also at the chirally broken case

$$
M\left(p^{2}\right)=\frac{2 \pi^{2} \gamma}{3} \frac{-\langle\bar{\Psi} \Psi\rangle}{p^{2}\left(\frac{1}{2} \ln \frac{p^{2}}{\Lambda^{2}}\right)^{1-\gamma}}
$$

where $\langle\bar{\Psi} \Psi\rangle$ is the chiral condensate and $\Lambda$ is the characteristic scale. To take both functions into account we use the following regularised version

$$
M\left(p^{2}\right)=\frac{2 \pi^{2} \gamma}{3} \frac{-\langle\bar{\Psi} \Psi\rangle}{\left(p^{2}+a^{2}\right)^{2 b}\left(\frac{1}{2} \ln \frac{p^{2}+c^{2}}{\Lambda^{2}}\right)^{1-\gamma}}
$$

We will use this function to fit our resulting quark propagator while we leave all quantities as free fit parameters.

\section{Results}

The results for the wave function renormalization and mass function are shown in figure 1 . We have given both functions in lattice and physical units. First of all we observe that the wave function renormalization is one within systematic errors (which are not given in the plot) [12]. In the mass function we observe an infrared enhancement which decreases for larger momenta. Applying the fit function (3.3) to our result we find the fit parameters given in table 1 . We see that $b$ is close 

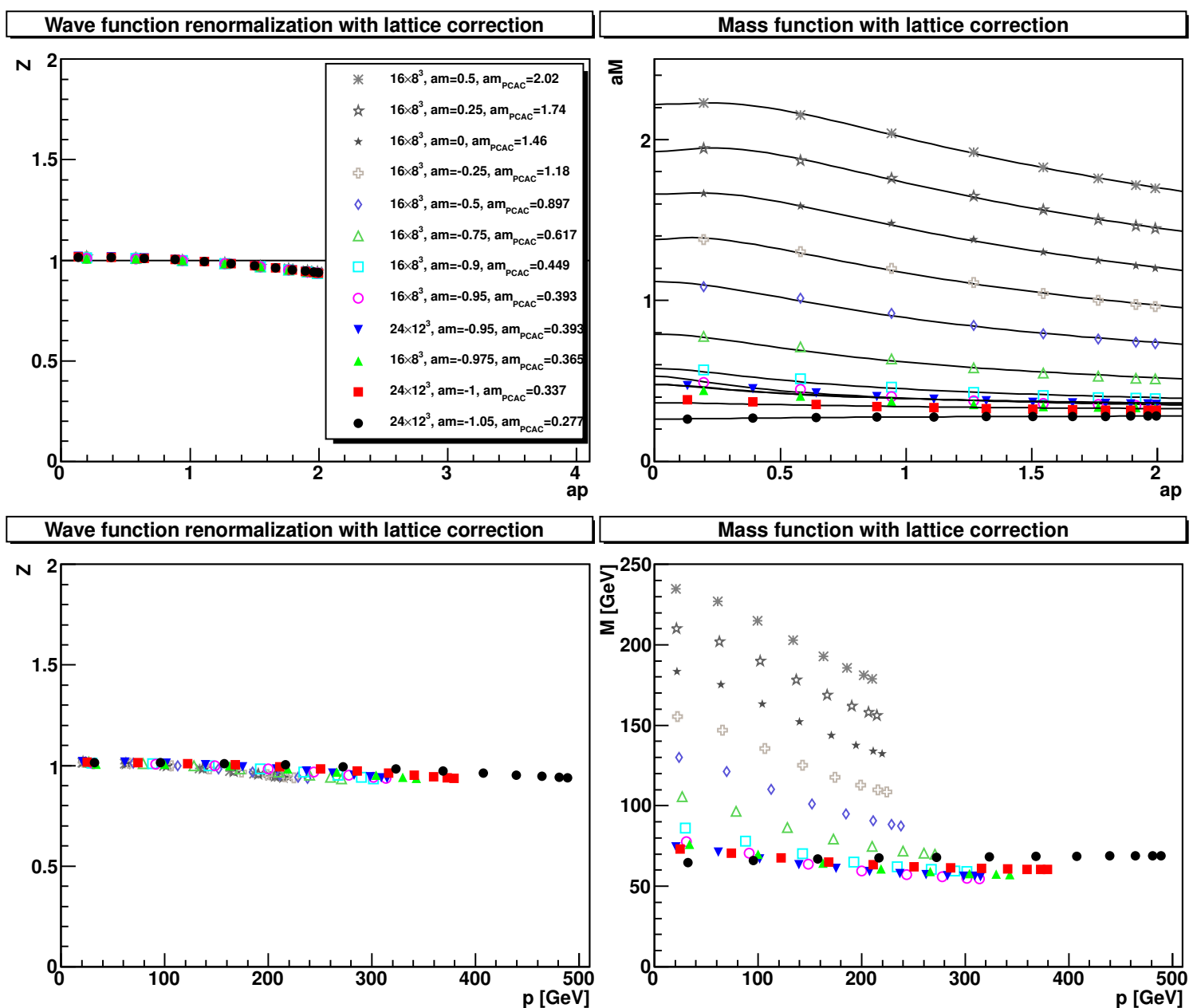

Figure 1: The wave function renormalization (left panels) and mass function (right panels) in lattice units (top panels) and physical units (bottom panels) for different masses and volumes. For the case of lattice units also fits are shown. Renormalization is performed at $a \mu=1$.

to zero for small quarks masses while $\gamma$ is close to one. Furthermore the ratio $M(0)$ to $M(\mu)$ is close to one within errors for the smallest quark masses. Both observations indicate that there is no spontaneous symmetry breaking for small quark masses. Following $[5,18]$ we would expect such a behavior. On the other hand this could also be a finite volume effect. Therefore to resolve this matter much more systematic investigations are required.

Another problem could be that the largest accessible momenta are not large enough with respect to all other scales. This leads to an influence of the renormalization in our results. For example using (3.1) as the fit form we find $\gamma$ to be in range $0.2-0.3$ which is in line with previous results, e.g. $[3,4,5]$. Comparing (3.1) with (3.3) we find $\gamma_{p}=1-\gamma$ which means that the anomalous mass dimension for the fit (3.3) is even closer to zero. This result suggest that also the fit form is a systematic error. 

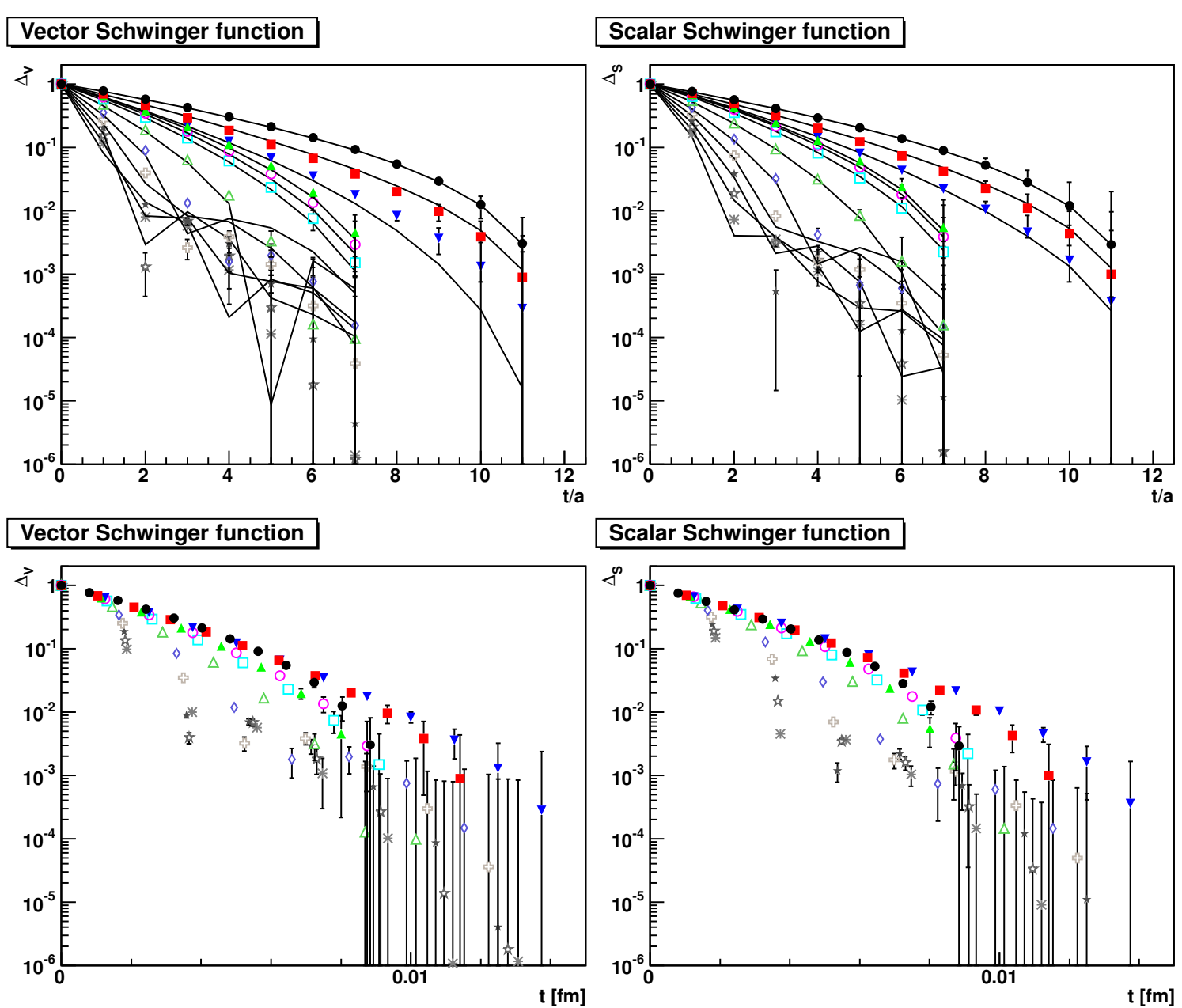

Figure 2: The vector (left panels) and scalar (right panels) Schwinger functions in lattice units (top panels) and physical units (bottom panels) for different masses and volumes. For the case of lattice units also fits are shown. Renormalization is performed at $a \mu=1$. The symbols are the same as in figure 1

\section{Schwinger Functions}

The Schwinger functions (2.3),(2.4) are shown in figure 2 in lattice and physical units. We can conclude from the bending of all curves that the techniquarks do not posses a positive-definite spectral function. We even observe zero crossings for larger masses. These observations imply that the techniquark does not belong to the physical state space, yet this is not enough evidence to claim that the techniquark is confined.

\section{References}

[1] J. R. Andersen et al.,Discovering Technicolor, Eur.Phys.J.Plus 126,81 (2011) [1104 . 1255 [hep-ph] ]

[2] S. P. Martin, A Supersymmetry Primer [hep-ph/9709356] 
[3] L. D. Debbio, B. Lucini, A. Patella, C. Pica and A. Rago, Mesonic spectroscopy of Minimal Walking Technicolor, PhysRev. D82, 014509 (2010) [1004.3197 [hep-lat]]

[4] A. Patella, A precise determination of the psibar-psi anomalous dimension in conformal gauge theories, PhysRev D86, 025006 (2012) [1204.4432 [hep-lat]]

[5] L. D. Debbio and R. Zwicky, Hyperscaling relations in mass-deformed conformal gauge theories, PhysRev D82, 014502 (2010) [1005.2371 [hep-ph] ]

[6] A. Cucchieri, A. Maas and T. Mendes, Exploratory study of three-point Green's functions in Landau-gauge Yang-Mills theory, PhysRev D74, 014502 (2006) [hep-lat / 0605011 ]

[7] L. D. Debbio, B. Lucini, A. Patella, C. Pica and A. Rago, The infrared dynamics of Minimal Walking Technicolor, PhysRev D82, 014510 (2010) [1004.3206 [hep-lat ] ]

[8] L. D. Debbio, B. Lucini, A. Patella, C. Pica and A. Rago, Conformal vs confining scenario in SU(2) with adjoint fermions, PhysRev D80, 074507 (2009) [0907.3896 [hep-lat ] ]

[9] L. D. Debbio, A. Patella and C. Pica, Higher representations on the lattice: numerical simulations. SU(2) with adjoint fermions, PhysRev D81, 094503 (2010) [0805.2058 [hep-lat]]

[10] R. Alkofer, W. Detmold, C. S. Fischer and P. Maris, Analytic properties of the Landau gauge gluon and quark propagators, PhysRev D70, 014014 (2004) [hep-ph/ 0309077 ]

[11] A. Maas, Gauge bosons at zero and finite temperature, Phys. Rept. 524 (2013), pp. 203-300 [1106.3942 [hep-ph]]

[12] D. August, A. Maas, On the Landau-gauge adjoint quark propagator, JHEP 1307 (2013) 001 [1304.4423 [hep-lat]]

[13] A. Maas, On the gauge boson's properties in a candidate technicolor theory, JHEP 1105, 077 (2011) [1102.5023 [hep-lat]]

[14] J. Fröhlich, G. Morchio, and F. Strocchi, Higgs phenomenon without symmetry breaking order parameter, Nucl.Phys. B190, 553 (1981)

[15] A. Maas, Bound-state/elementary-particle duality in the Higgs sector and the case for an excited 'Higgs' within the standard model, Mod.Phys.Lett.A, Vol. 28, No. 28 (2013) 1350103 [1205. 6625 [hep-lat]]

[16] ATLAS Collaboration, Combined search for the Standard Model Higgs boson using up to $4.9 \mathrm{fb}-1$ of pp collision data at sqrt $(s)=7 \mathrm{TeV}$ with the ATLAS detector at the LHC, Phys.Lett. B710 (2012) 49-66 [1202.1408 [hep-ex]]

[17] CMS Collaboration Combined results of searches for the standard model Higgs boson in pp collisions at $\operatorname{sqrt}(s)=7 \mathrm{TeV}$, Phys.Lett. B 710 (2012) 26 [1202.1488 [hep-ex] ]

[18] L. D. Debbio, R. Zwicky, Scaling relations for the entire spectrum in mass-deformed conformal gauge theories, SHEP 10-28 CPR 2010-38 [1009.2894 [hep-ph] ] 